\title{
Cognitive-Reminiscence Therapy for Young Adults with Depressive Symptoms: Clinical Observations, Challenges, and Recommendations
}

\author{
Hallford, D. J. ${ }^{1,2}$ \& Mellor, D. ${ }^{1}$ \\ ${ }^{1}$ School of Psychology, Faculty of Health, Deakin University, 221 Burwood Hwy, Burwood, Victoria 3125, \\ Melbourne, Australia \\ ${ }^{2}$ Corresponding Author, School of Psychology, Deakin University, 221 Burwood Hwy, Burwood, Victoria \\ 3125, Melbourne, Australia, Phone: + 6139251 7777, Email: david.hallford@deakin.edu.au
}

PLEASE CITE AS: Hallford, D. J., \& Mellor, D. (2018). Cognitive-Reminiscence Therapy for Young Adults with Depressive Symptoms: Clinical Observations, Challenges, and Recommendations. Unpublished manuscript. 


\begin{abstract}
Cognitive-reminiscence therapy is a novel intervention for depression in young adults, and currently no specific clinical guidance exists for its use in this age group. The aim of this article is to disseminate our experiences from a recent clinical trial in which we engaged in cognitive-reminiscence therapy (CRT) with young adults for the purpose of treating depressive symptoms. Discussion is presented in terms of clinical observations that were made during treatment, specific challenges that arose, and recommendations for implementing this therapy with young adults. Firstly, a conceptual overview and rationale for CRT is provided along with an outline of the treatment protocol that was used. Then, a framework for undertaking therapeutic reminiscence work at different levels of analysis is presented, and comment is made on the rationale for, and the process of, eliciting specific memories. Common life events and issues that emerged as the foci of reminiscence with young adults in our trial are reviewed. Following this, the process of orienting to the mechanisms of change in CRT is discussed. Finally, several clinical issues and insights that emerged during the provision of this therapy are presented: contextualising emerging adulthood as a time of transition, creating continuity across individuals' lives, the process of reflection on reminiscence, and homework adherence.
\end{abstract}


In recent times, the development and evaluation of reminiscence-based psychotherapeutic interventions for depression has become an increasingly active area of research. Meta-analytic evidence has indicated that reminiscence-based interventions, in general, are highly effective in treating depression (Pinquart \& Forstmeier, 2012), with structured interventions with explicated theories regarding change processes significantly more so. One such therapy, referred to as cognitive-reminiscence therapy (CRT; Cappeliez, 2002), strategically utilises two particular types of reminiscence. These are integrative reminiscence (the use of memory to provide meaning and continuity of the self, and to reconcile past negative experiences; Wong, 1995) and instrumental reminiscence (drawing on past experience in planning, coping, and solving problems; Wong, 1995). During CRT, therapists draw on cognitive models of depression and cognitive therapy techniques (Beck, Rush, Shaw, \& Emery, 1979), as well as stress and coping models of depression (Billings \& Moos, 1985) and problem-solving techniques (Nezu, 2004) for use within therapist-guided integrative and instrumental reminiscence. A series of clinical trials have demonstrated CRT to have typically large effects in ameliorating depressive symptoms in older adults (for a review of these studies see Hallford \& Mellor, 2013a).

Consistent with the distinct paucity of research into reminiscence-based interventions with young adults, no clinical guidance currently exists on the use of CRT for depression within this age group. This is perhaps unsurprising given that reminiscence therapies have been historically conceptualised as relevant only to older adults. Reminiscence-based therapies have long been used with older adults in the context of successful ageing, death preparation, and the development of ego integrity (Butler, 1974). 
Indeed, older adults perception of less time in life ahead of them than behind them (Carstenson, 2006) is likely to give rise to the psychosocial goals of achieving an integrated and positive sense of identity, and belief that one's life has been significant and meaningful (Erikson, 1980). The notion of learning from one's past, and revisiting or reappraising experiences to promote positive self-worth and self-confidence also emerge as central aims of this approach to therapy.

Unfortunately, the potential utility of reminiscence-based therapy in other stages of adulthood has, to date, been largely neglected. Recently, we argued that various lines of developmental, cognitive, and clinical evidence converge to indicate that this form of intervention may well be appropriate and useful for younger adults (Hallford \& Mellor, 2013a). Young adults have amassed an array of experiences as they developed through childhood, adolescence, and emerging adulthood. These experiences provide a wealth of content to draw on in reminiscence-based therapy, and with which to achieve the same therapeutic aims as with older adults (although typically not preparation for impending mortality). More specifically, young adults are faced with a significant amount of developmental transitioning as they move from adolescence into adulthood. These changes occur across a range of domains, such as social, occupational, and that of the self-concept. Unsurprisingly then, self-concept clarity and self-continuity is relatively more important for adults of this age (Bluck \& Alea, 2008, 2009). This stage of life also sees the emergence of a coherent narrative identity informed by the developed ability to use reflection and reasoning with autobiographical memory to connect one's life stories to a sense of self (McAdams, 2001). CRT aims to promote meaningful self-continuity, and negotiate a more positive narrative identity congruent with the self as being capable 
and worthwhile by using specific therapeutic techniques to combat depressogenic patterns of thinking. Interventions such as CRT target developmentally-relevant issues for young adults. The failure to manage transitions during this time may well have significant implications for future functioning, such as further depressive episodes of longer duration and higher severity (Korten et al., 2012; Zisook et al., 2007). Helping a depressed or at-risk young person navigate this transition or reconceptualise it in a healthy way is necessary to facilitate successful adaptation in adult roles. Moreover, young adults that are depressed are unlikely to effectively utilise their past experiences, given their relatively maladaptive use of specific autobiographical memories (Williams et al., 2007) and construction of life narratives (Fromholt, Larsen \& Larsen, 1995; Habermas, Ott, Schubert, Schneider, \& Pate, 2008). CRT provides the means to adaptively access and draw on personal resources they may not otherwise.

On the basis of this rationale, we have conducted a trial of CRT with young adults aged 18-25 years that aimed to investigate its potential in ameliorating depressive symptoms (full trial results forthcoming; see Hallford \& Mellor, 2013b for a study protocol). Pilot data indicates that this intervention is effective (Hallford, 2014), with large effects with regards to the reduction of depressive symptoms, and the majority of participants either recovering or reporting clinically-significant reductions in these symptoms. Further, young adults who received cognitive-reminiscence treatment perceived it to be a positive, relevant, and useful therapy (Hallford, Mellor, \& Burgat, under review).

Given the novel use of reminiscence-based therapy in this demographic, our experience conducting the trial provided us with a unique opportunity to gain insights 
into the process of delivering this therapy with young adults. In this article, we draw on these experiences to disseminate clinical observations that were made, identify specific challenges that arose, and provide guidance in implementing this therapy with young adults. Initially, a framework for undertaking therapeutic reminiscence work at different levels of analysis will be presented, and comment made on the rationale for, and process of, eliciting specific memories. Common life events and issues that emerged as the focus of reminiscence with young adults in our trial will be reviewed. Following this, some discussion will be presented on the process of orienting to the mechanisms of change in CRT. Finally, several clinical issues and insights that emerged during the provision of this therapy will be then discussed: contextualising emerging adulthood as a time of transition, creating continuity across individuals' lives, the process of reflection on reminiscence, and homework adherence. To provide a context for subsequent sections, a description of the treatment protocol used in our trial will first be presented.

\section{A Protocol for Cognitive-Reminiscence Therapy}

The basic format and content of the cognitive-reminiscence intervention for young adults was adapted by Hallford (2013) from a manual developed and evaluated by Watt and Cappeliez $(1995,2000)$ in their work with older adults. Several key changes were made in adapting this therapeutic approach for use with young adults: integrative and instrumental reminiscence were used in conjunction rather than in separate protocols for therapy (as per Cappeliez, 2002); therapy was individual rather than group-based; and adaptations were made to questions used in sessions and on homework sheets to be ageappropriate for young adults. 
In total, six 60-minute weekly sessions are provided. In the first session the individual's current depression-related issues are briefly reviewed, brief psychoeducation is provided on cognitive and stress and coping models of depression, and autobiographical memory (specifically integrative and instrumental types of reminiscence), and the process and aims of the intervention are discussed explicitly. A problem list is then generated for use in subsequent sessions. Memories related to the first reminiscence topic of turning points are elicited, and among these several are identified as being relevant to integrative and instrumental reminiscence, and to be discussed further. These memories are then elaborated on, with the therapist ensuring that sufficient details are elicited to promote memory specificity and inform therapeutic work. Cognitive therapy techniques (Beck et al., 1979) are used while engaging in integrative-type reminiscence to promote cognitive distancing and reattribution, emphasis positive and more balanced evaluations of experiences, reinterpret negative memories to be more rational and adaptive, appraise self-worth and meaning in life in accordance with internal standards, and foster a sense of continuity and a positive narrative identity. On engaging in instrumental-type reminiscence, a stress and coping framework (Billings \& Moos, $1985)$ is used to conceptualise past experiences and promote the appraisal of stressors as challenges rather than threats, recognise and develop coping resources, and promote awareness of prior constructive problem-focussed coping. A summary of the reminiscence work is then provided and feedback is elicited from the participant on their impressions of what has been discussed, what they have learned, and any changes that may have occurred for them. Homework sheets containing information about problemsolving, and questions designed to elicit reminiscence related to the next week's topic are 
provided. The remaining sessions follow a procedure of reviewing homework for the week, identifying memories to elaborate on and engaging in therapeutic work with these memories, reflecting on this reminiscence, and then utilising a problem-solving framework to choose solutions to identified problems to implement over the next week. In the last session, the therapist provides an overview of the work completed over the course of the intervention, and the participant is invited to provide feedback and reflect on their experience. Over the six sessions, a different topic is used each week to stimulate memories as a basis for therapeutic reminiscence work: turning points, family life, significant/important activities in your life, loves and hates, stressful experiences, and meaning in life.

As might be garnered from the above description, reminiscence work is likely to involve discussion of specific past experiences as well as broader personal narratives. On the basis of our experience delivering this intervention, we have formulated a heuristic for working with reminiscence at different levels of analysis. This will now be described.

\section{Levels of Analysis in Therapeutic Reminiscence Work}

Given that therapeutic reminiscence work involves reviewing and integrating memories, a three-level model of analysis may facilitate conceptualisation of this process for therapists' wanting to use this approach (see Figure 1). The first level consists of the specific factual details, emotions, and thoughts that are associated with autobiographical memories. This level relates to the what, where, when, how, and why of memories, as well as how the person felt and what they thought during specific experiences. For example, where and when the person experienced a specific stressor in their life, facts 
regarding the coping process and outcome, associated feelings (e.g. pride, guilt, disappointment, excitement), and their beliefs about themselves in the context of that situation at the time. The second level relates to the perceived significance of the event or experience in individuals' lives, and how it might serve to define the self. For example, whether the memory was an illustration of how they had overcome a challenge, whether it was a meaningful event for them and why, how they appraised the overall outcome, how it relates to their self-concept (e.g. it increased their self-confidence at the time), and how it might have changed their life. The third, and most macro level, relates to sequences of events and experience in terms of a broader narrative. On this level, memories are integrated to form an overarching story of the self with unity, purpose, and an internally-derived standard of worth. As McAdams (2001) suggests, broader life narratives must integrate sometimes conflicting experiences, roles, and relationships across time, and go beyond biographical details to appropriate aspects of experiences, and imaginatively construct stories about the self that vivify and integrate life. These stories are used to define the self in a way that is rational, meaningful, and fosters a positive selfconcept. For example, on reviewing work over the course of sessions, one individual in our trial was able to re-construe their social life as being one of learning from challenges and successes, rather than failure as the result of a character deficit. Although his earlier schooling years involved some bullying and anxiety about integrating into social groups, these experiences led him to form strong bonds with others in marginalised groups, and value their solidarity. These secure friendships helped him build confidence over time, and he learned better ways of relating to others, as well as what he did and did not value in friendships. Over time, he developed methods of building and maintaining stable and 
meaningful relationships, even during times of change in his life (e.g. starting a new job or when friends moved away). Recognition and integration of these experiences, including how he had been valued by others and the social skills he had built, helped foster the belief that he was able to be socially effective, and increased his hope and confidence that he would continue to be.

Specific therapeutic techniques can be implemented at various levels. Examples of this are cognitive reappraisals of self-beliefs in a specified situation (first level) or across a range of experiences (third level), or identification of a useful problem-solving strategy used in a specific situation (first level; e.g. scheduling study time before exams) or a style of coping used across a series of experiences (third level; e.g. seeking support from others). Continuity is a core aim here (see below), through weaving together patterns of first and second levels of analysis into a broader positive and cohesive life narrative. An example of this is provided in Figure 2 to illustrate how separate foci of reminiscence can be collated to form a superordinate narrative to reflect on (for further reading see Singer, Blagov, Berry, and Oost [2013] who provide a more detailed account of the components involved in a narrative identity). In Figure 2 it can be seen that the first level involves details of three distinct coping experiences, the second shows the significance of these experiences in the person's life, and the third level draws together these memories to form a common theme of being capable and overcoming challenge.

In the context of depression, individuals' beliefs about themselves and their life stories are likely to be biased by negative thinking styles. Through this therapeutic approach they are engaged in the process of collaboratively reviewing, evaluating, and 
integrating their life and experiences "from the ground up". Issues that emerged in our trial relating to the elicitation of memories will now be discussed.

\section{Challenges in Eliciting Memories, and Why this is Important}

It is well established that clinically depressed individuals report less specific and more overgeneral autobiographical memories (Liu, Li, Xiao, Yang, \& Jiang, 2012), which has been associated with impaired problem-solving and a reduced ability to use past events and experiences to accurately predict future events (Williams et al., 2007). This overgeneral style of memory retrieval is likely to create potential barriers in the context of reminiscence-based therapies. Further, it plays a modest, but significant role in maintaining depression (Sumner, Griffith, \& Mineka, 2010). Fortunately, a growing research literature indicates that increasing memory specificity through simple practice in retrieving and elaborating on autobiographical memory, even without additional guidance or psychotherapeutic intention, can significantly reduce depressive symptoms (NeshatDoost et al., 2013; Raes, Williams, \& Hermans, 2009; Serrano, Latorre, Gatz, \& Montanes, 2004; Serrano Selva et al., 2012), as well as increase problem-solving skills and reduce hopelessness and negative rumination (Raes et al., 2009). In one study with adolescents, memory specificity practice produced large reductions in depressive symptoms, with increases in memory specificity also predicting changes in depression at follow-up (Neshat-Doost et al., 2013). These recent findings show that the act of eliciting specific memories through reminiscence-based therapy is likely to be an intervention for depression itself, even before any further psychotherapeutic technique is utilised. Given 
that details of experiences are also needed to effectively review memories and use therapeutic techniques, the moral of the story is that specifics count.

It was our experience during our trial that young adults often failed to elaborate on autobiographical memories of their own volition, and found it challenging at times to remember details. Reasons stated for this included forgetting, trouble concentrating, and difficulty retrieving details that were deemed to be of significance. As might be predicted, overgeneralisation was also a common factor in this process. For example, individuals might construe high school as a single event instead of a long series of related but separate experiences over the course of many years, or refer to a past relationship as being wholly negative and minimise positive aspects on the basis of a hurtful ending. In the event that individuals were unable to initially identify memories to discuss, we found that assisting them in the task by suggesting possibilities based on common experiences in this stage of life (see below) was useful. Once memories are chosen individuals can be probed for further factual details with focussed questions such as "Who was there?" "What were the sights, sounds, and smells?" "What lead up to this?" "How long did that last?" "What happened next?" Being creative with this process, and explicit about the importance of it in the context of the therapeutic work, was found to be helpful.

Eliciting associated emotions and thoughts was an important next step, so as to draw out perceptions, attitudes, and personal meaning. Questions such as: "How did that make you feel?" "What was the impact of that on you?" "How important was that to you?" "What was your view of that?" "Can you remember what you were thinking at the time", were found to be useful. This process helped to draw out feelings, beliefs and appraisals of the experience and the self that were either adaptive (and therefore fostered 
and reinforced) or negative (and therefore potential candidates for review and reinterpretation). Asking about factual details of memories prior to moving on to related emotions and thoughts was a useful sequence of questioning, so as to avoid appearing to trivialise feelings by reverting back to details that might be considered less meaningful to individuals.

As well as ensuring that reminiscence incorporated specific details of the event, experience, relationship etc. the quality of the autobiographical memories that were concentrated on were found to be of vital importance. The content, scope, and personal meaning aspects of these memories are important factors in the effectiveness of subsequent psychotherapeutic work. A rule-of-thumb pertaining to memories being of integrative or instrumental relevance, and of moderate-to-high significance to individuals was found to be useful. Individuals may implicitly or explicitly indicate that memories are of importance to them, or alternatively this can be directly asked by the therapist. Although guiding questions on the homework sheets typically helped direct individuals to appropriate memories, they were not always a failsafe method for this purpose, and the process was typically quite collaborative in nature. Matching reminiscence content with the individuals' presenting depressogenic beliefs is also recommended. For example, individuals in our trial who felt pessimistic about their current abilities were guided to discuss past experiences of success, or where there was a recurrent theme of feeling unworthy, an emphasis was put on times when individuals were valued by others and appreciated or were recognised for an achievement.

It should also be noted that on discussing meaning in life in the last session, we found a tendency for individuals to find this concept somewhat abstract. One method to 
facilitate this reminiscence effectively was by referring to personal values and ways in which individuals wanted their life to be (e.g. being a good friend or being artistically creative) and then using their responses to identify past experiences in which these values and ways of living were being realised (e.g. a time when they helped a friend with something important, or a memory of a project or performance they were proud of).

\section{Life Events and Common Developmental and Psychosocial Issues in Emerging Adulthood}

While we suggest that this type of intervention is likely to be appropriate for adults of all ages, the individuals who received CRT through our trial were between 18 and 25 years of age, and therefore some comment on reminiscence topics that emerged within this cohort is warranted. Individuals referred predominantly to experiences in their life associated with adolescence and emerging adulthood, and less so to those associated with childhood. A selection of prominent themes that emerged is provided in Table 1. This is by no means an exhaustive list, but rather events and experiences that were commonly discussed, and were found to be useful for therapeutic purposes. The relationship between issues in columns can be seen, and how they might cohesively relate to one another. For example, moving out from the family home usually led to changing relationships with parents and siblings (or vice versa), and starting university was usually followed by challenges faced in completing assessment which resulted in growing specialised knowledge. In most sessions, reminiscence would cover several of these interconnected issues and transverse different domains. Although each individual can be understood as having idiosyncratic positive and negative experiences in their 
development, it can be seen that each of these has the potential to be discussed as being meaningful, and contextualised as a growth experience or a challenge that was overcome. Lessons learned in orienting to the proposed mechanisms of change in CRT while reviewing such memories will now be discussed.

\section{Therapist Orientation to the Underlying Mechanisms of Change}

An inherent risk in discussing a wide range of domains across the lifespan is that the aim of therapeutic work may become too diffuse, sessions may lose focus, and reminiscence might become more descriptive rather than purposefully analytical in nature. Fortunately, therapists can orient themselves towards specific psychological constructs related to change that are embedded in individuals' reminiscence, and manifest through the process of review. Theoretical formulations of CRT (Watt \& Cappeliez, 1995, 2000) and adaptive reminiscence (Wong, 1995), as well as empirical evidence from basic and clinical outcome research (Cappeliez \& Robitaille, 2010; Hallford, Mellor, \& Cummins, 2013; Korte, Cappeliez, Bohlmeijer, \& Westerhof, 2012; Westerhof, Bohlmeijer, van Beljouw, \& Pot, 2010), have provided signposts to psychological constructs implicated in reductions in depressive symptoms, with three garnering relatively more support to date: self-esteem, self-efficacy, and meaning in life.

At the heart of CRT is the aim of promoting a positive self-concept, incorporating a sense of worth and competence throughout the lived life. Therefore, increasing selfesteem and self-efficacy through the discussion of experiences that promote positive selfevaluations in these terms is likely to be of benefit. In regards to self-esteem, experiences that promote a positive attitude towards the self, such as those related to feelings of pride, 
worthiness, and self-respect are likely to be beneficial in balancing negative appraisals of the self that typically accompany depression. We found there were typically a range of memories of significant happenings, relationships, achievements etc. that could be retrieved and reviewed to contribute to a stronger sense of positive self-esteem.

Therapists can work to ensure these types of memories are chosen for further discussion at the start of sessions, prompt individuals to focus on them if they are not retrieved as part of reminiscence homework, or find positive aspects of experiences that individuals initially perceive as wholly negative. For example, in our trial one individual lamented leaving a paid position in a professional sports team, and viewed this as a sign of personal failure. This experience was drawn out in more detail, with other moderating factors considered, such as apparent lacklustre view of the team itself, the drawbacks of geographically moving to be part of the team, and the competing interests he held at the time such as returning to study. The appraisal of "not being good enough" was contrasted with other ways of understanding these events, such as his achievement in getting into the team in the first place, and moments of pride he experienced during his time in the team. In general, past events do not lose their significance simply because things have changed, and positive experiences remain positive experiences, regardless of what subsequently occurs. We found that highlighting this helped individuals to identify and value the positive aspects of experiences. Clearly, not all negative experiences can be alternatively construed, but can typically be embedded in the broader, more balanced story of people's lives, which can moderate their impact on self-esteem.

Self-efficacy was most clearly focused on during instrumental reminiscence. Discussing occasions when individuals overcame challenges, achieved important goals, 
and coped with stress provided excellent opportunities to bolster their sense of selfefficacy and approach-oriented coping in the present. Fostering the recognition of these experiences was central to avoiding them being marginalised and "swept under the carpet" in the context of depression. Further, identifying specific strategies that have been used to overcome previous challenges was found to help individuals' to utilise them in the present. In our trial, one individual found himself needing to conduct research into organisations in which he had applied for jobs in a very short period of time. By drawing on discussion from a previous session relating to how he had coped with exam stress in high school he was able to identify techniques he could use in the present to maximise his productivity (e.g. finding a quiet place he would not be interrupted, working in the mornings when he found he was most alert etc.)

As a general observation, young adults in the trial appeared to take their recent experiences to be the most important predictor of their coping ability in the present and into the future. This neglect of prior coping experiences may well be a product of several factors, such as a bias for overgeneralised or negatively-valenced memory due to depression (Williams et al., 2007), or an age-related bias of present and future timeperspective (Lang \& Carstensen, 2002; Webster \& Ma, 2013). However, in light of discussing previous experiences that contradicted a global appraisal of low self-efficacy, individuals found themselves becoming more ambivalent, or optimistic about previously negatively-valenced predictions. For example, one individual, after recalling and reviewing a string of challenging past circumstances highlighting her methods of adaptive coping in situations, remarked, "I can see now that sometimes I sell myself short". This opened up the opportunity to examine more experiences that contradicted her 
current negative view of herself, and became a useful anchor for further construction of a view of the self as being capable and competent.

The concept of personal meaning in life is explicitly addressed in the final session of CRT for young adults with depression; however, therapists can identify and discuss experiences that relate to meaning at any stage of the intervention. What constitutes a sense of meaning in life can differ significantly for individuals, and may encompass living by certain values, moving towards a particular goal, or having a sense of the interrelatedness of experiences. Young adults in the trial, predominantly construed personal meaning as having goals in life, with examples such as getting into a university course or working in a helping profession. Anchoring this meaning to individuals' sense of self and decision-making proved to be a valuable means by which to strengthen the perception of purpose in their life. Discussing the emergence of this meaning, how it had been fostered over time, and its significance in the present and for their future helped to weave self-continuity and the sense of this meaning as providing guidance and cohesion in their life. By increasing awareness of the causal and thematic interrelatedness of experiences, and their relevance as part of a life story, therapists can foster an implicit sense of meaning, purpose, and agency. Young adults in our trial reported that this process helped them feel connected to a self-determined path that their life was taking, and made them feel more hopeful and optimistic about their future (Hallford et al., under review).

It is worth noting that meaning can be derived from experiences that, in isolation, might otherwise be perceived as purely negative. In this sense, the therapist assists the individual in contextualising and transforming the meaning of memories. For example, 
through guided reminiscence one individual established that a period of intense family conflict during his adolescence provided insight into the problems at the core of their inability to get along with each other. Further after hitting "rock bottom" with his family, he had also learned to more strongly value his relationships with family members and could identify the positive effects they could have on his life.

Delineating these psychological constructs may be, in part, an exercise in posthoc analysis. In reality, discussions of memories in session typically involved content relevant to several of them. A brief outline of one session may serve to illustrate this. One individual, who had previously expressed feeling inadequate in regards to his ability to make friends, elected to review the process of leaving his family home to attend university in a new city during a session initially focussed on family life. After discussing the process of transitioning from dependency to autonomy, he reported how he had been lonely on arrival in his new city home. His experiences in facing this challenge were discussed, and he reported how he had spent a couple of years establishing a friendship network through university, becoming involved in several hobby clubs related to his interest in performing arts, and eventually developing acquaintanceships into friendships. By reviewing these efforts, he was able to identify evidence of his competence in becoming independent and making new friends, increase his self-worth through the interest that others had taken in him and the social connectedness he had built, and reinforce the growing meaning and centrality of being artistically creative in his life.

Explicitly orienting individuals to the aims of the reminiscence work from the outset was useful, and there was no obviously apparent gain in keeping the objectives implicit or indirectly communicated. Typically, individuals were motivated to discuss 
positive experiences, as well as to reinterpret those that had been plaguing them and maintaining depressive symptoms. Given the inherently personalised work of reminiscence therapy, the content that is discussed is highly relevant for individuals.

Given the scope for discussion, therapists may find themselves steering off-course at some point during sessions. We found that a quick evaluation of whether or not the discussion is focussed on any of the aforementioned constructs was a useful heuristic. Additional questions that therapists might ask themselves could be, 'Would discussing this memory be likely to highlight a positive experience or attribute of this individual?, "Will it have a positive effect in some way?", "What might this person be able to learn from this?", "Is there any way this memory could be adaptively reappraised?", and "Is this person overlooking certain parts of this experience that might be beneficial for them to discuss?"

\section{Emerging Adulthood as a Time of Change}

Emerging adulthood marks a critical period of psychosocial development in life (Arnett, 2000). The transition from adolescence to adulthood is accompanied by increased vulnerability to depressogenic psychosocial stressors (Hammen, 2005), at a time when individuals are only beginning to become self-sufficient, develop their socialcognitive maturity, and understand themselves and others in more sophisticated ways. Given this, it is perhaps unsurprising that the total number of significant life events, and specifically those that are negative, uncontrollable, and occurring in social role areas of school, education, family, work, and living conditions, predicts the onset of depression in young adults (Friis, Wittchen, Pfister, \& Leib, 2002). Unfulfilled expectations regarding 
perceived markers of adulthood, and unattained goals during this transition to adulthood can have negative social and psychological consequences, and accordingly, are also risk factors for depression (Hammen, 2005; Mossakowski, 2011). This can result in young adults feeling a sense of inferiority, perceived distance between the self and goals at this stage of life, and subsequent concerns about meeting expectations of themselves now and in the future (Kuwabara, Van Voorhees, Gollan, \& Alexander, 2007). Congruent with this, many of the individuals who received CRT in our trial expressed depressogenic beliefs relating to having failed at effectively launching their next stage of life in adulthood, making poor choices during or following high school, and feeling "lost" and without purpose.

Framing the young person's stage of life as one of beginnings, explorations, learning, and change was found to be a useful method in creating flexibility around the narrative of their life, and the context in which perceived disappointments or "wrong turns" occurred. For example, several individuals perceived leaving university as a failure on their behalf, even in instances where they had not failed in terms of their academic results. Framing their time at university as a means of exploring avenues of interest, one that has helped them clarify what it is that they are or aren't interested in pursuing, was found to be a useful approach to take. The therapist may posit that individuals cannot expect to be certain about how their plans will turn out, or of the outcomes of their efforts. Although decisions may have been made in good faith at the time, they cannot guarantee that they will enjoy the pathways that they set off on in young adulthood. Stating this explicitly, gently challenging the young adults' ability to have reliable preconceptions or expectations of these experiences (e.g. "was it possible for you to 
know exactly what it would be like?"), and framing it as a learning experience were useful strategies to reframe their appraisal of failure and address associated feelings of guilt. These experiences can also be framed as helping them to be better informed to make future choices. Assisting in reaffirming or reappraising decisions individuals had made in life as intentioned choices was found to bolster individuals' perceptions of an internal locus of control, and help them rely more on an internally-derived standard of worth. In several instances, broader challenges were used to frame their stage of life, such as "maybe this is a time when you are still working these things out and still finding out who you are, and what you want. Can that be an okay stage to be at?"

Arnett (2004) proposes that emerging adulthood is a distinctive time in life due to it being the age of identity explorations, age of instability, the self-focussed age, the age of feeling in-between, and the age of possibilities. The adoption of this view by therapist and client alike proved to be a useful lens through which to construe individuals' choices and the narrative of their life to date. Several individuals achieved "sudden gains" of clinically significant reductions in depression through this method, signalling the centrality of this in their life narrative, and their experience of depression.

\section{Creating Continuity between Past, Present, and Future}

Continuity in the narrative identity was perceived as a major factor in individuals' depression at this life stage, and concerns about "where my life is headed" were particularly common from the outset of intervention. Fostering this continuity involved temporally linking experiences across the young adults' life to identify progression, change, and the influence that earlier experiences have subsequently had. Further, the 
significance of this story to the individual can be discussed and elaborated on, with events and experiences integrated to form a more cohesive and meaningful story. Noting that some things stay the same, while other things change was a useful way to frame these discussions when collaboratively integrating sequences of events over time. This helped individuals to recognise that "who they are" and "what their life story is", is a fluid concept that they have the capacity to create, change, and revise over time. In regards to the individual discussed above who had lost his position in a professional sports team, this event had the subsequent effect of pushing him to explore other interests and talents that he had, opening up other further possibilities for him and eventually leading to an interest in studying social sciences. Whilst the initial loss had an inherently negative emotional resonance, it could be more helpfully re-construed as also being a meaningful turning point and a new chapter in his life story.

It was common for individuals in our trial to be surprised at what they learnt from their past and the relevance of past experiences to current circumstances. Typically, individuals expressed benefiting from perceiving how intentional behaviors and motivations from their past had lead up to their present circumstances and how they themselves had driven this continuity. In addition to fostering continuity in the integrative sense, part of the cognitive-reminiscence approach involves a drawing on previous coping to inform a brief but concerted focus on present difficulties with problem-solving techniques. Creating continuity between past, present, and future, and fostering a "you have coped then during adversity, which hints that you are equipped to cope now" attitude was useful and tended to promote engagement and optimism towards problemsolving. Discussion of memories that related to competence and approach-coping 
behavior served as good tools to broadly promote individuals' self-efficacy and, in turn, their perceived control and willingness to attempt to solve current issues. These memories provide "hard evidence" for individuals that they are active and capable agents. Further, specific coping strategies that have been discussed in session were typically drawn on to assist in the problem-solving work in the present. One individual discussed her passion for playing the flute when she was younger and how this was an outlet for personal expression, as well as it bringing structure to her life through regular rehearsal sessions. On discussing solutions to one of her current problems, which was feeling aimless, re-engaging with playing the flute, with all the goodwill and meaning it had garnered in prior discussions of it, was identified as a possible course of action.

\section{Reflecting on Reminiscence and Gaining Feedback}

The importance of reflective discussion is made explicit at the beginning of the first session. However, promoting this throughout sessions and encouraging individuals' to explore the meaning of experiences or ways of viewing them was important, as was the meta-cognitive awareness of the process of reflection. For example, one individual discussed the transition from primary school to high school as being difficult, recalling the anxiety she experienced around starting at a new school and feelings of vulnerability and isolation. Through a process of reflection, and questions such as "What did you have to do to meet that challenge?", and "What did you learn from that?", she was able to identify and incorporate other aspects of this experience that involved having courage in taking risks and engaging with others, overcoming her anxiety, and developing her social skills. This process imbued the otherwise negative memories with a sense of having 
overcome adversity to some degree and the development of personal coping resources, both of which were linked with an eventual outcome of increased social connectedness. On reviewing this discussion at the end of the session, she was able to recognise the positive elements of this experience (reflection), and when prompted for feedback made brief comment on the helpful process of "seeing things in a broader context" (metacognitive awareness of the process of reflection). Likewise, during the overview of therapeutic work in the last session of therapy, making explicit the changes in perspective over sessions is likely to promote individuals' future ability to reflect, and to consider more helpful ways in which they might view themselves and their lives.

\section{Homework Adherence}

While not all reminiscence-based therapies involve between-session tasks, in CRT individuals are asked to complete homework sheets designed to elicit memories and associated details on the topic to be discussed in the following week. These homework sheets are beneficial as they promote adaptive reminiscence between sessions, increase memory specificity (see above), and save time within sessions. In our trial, individuals found the worksheets to be a good primer to stimulate reminiscence between and within sessions, however, a significant proportion of individuals neglected to complete them as directed between sessions (Hallford et al., under review). Non-compliance resulted in having to reallocate time away from other therapeutic activities, and made eliciting, drawing out, and reflecting on significant memories in session more difficult. In these cases, the initial autobiographical memories that were elicited sometimes lacked significant personal meaning in the context of their broader narrative identity. Individuals 
also tended to draw from current events that had strong emotional resonance for them, which somewhat compromised the opportunity for cognitive distancing, seeing events and experiences in the broader context of their life, and balanced reappraisal. Given this, it is suggested that the importance of setting aside time to complete these questions (which individuals reported as not taking very long) be communicated very clearly from the outset, and in the event of non-compliance be reiterated along with the rationale for completing them.

\section{Concluding Remarks}

Therapists may note the various frameworks that CRT explicitly (cognitive therapy, problem-solving therapy) and implicitly (solution-focussed, and strengths-based approaches) draws from, as well as the kinship with narrative approaches to therapy which explore alternative life stories (White \& Epston, 1990), without the emphasis on social constructionism. One way of conceptualising a CRT approach is as a method of guided reminiscence that flexibly utilises therapeutic techniques to draw on individuals' personal resources through their historical experience, reappraise or reconstruct experiences otherwise viewed as negative, and adaptively construct their broader life narratives.

It might be hypothesised that particular presenting issues within the context of depression might serve as indications for the use of CRT, such as issues with identity moratorium, transitioning into different roles or circumstances, existential anxiety, or feeling a lack of meaning or purpose in life. Young adults who signal a desire for selfreflection on their life might also be a "good fit" for this approach. Given that normative 
psychosocial development at this time is likely to involve significant life "turning points" and transitions into social roles, we observed that some or many of the aforementioned issues were embedded in presenting concerns, or emerged during therapeutic work in our trial. However, these observations remain anecdotal, and further systematic clinical investigation is needed to understand their association with process and outcomes in CRT. We found that regardless of the perceived aetiological or maintaining factors of depression, the therapist and client could collaboratively draw upon a wealth of useful personal resources from clients' memories of previous experiences, and what they had learned about themselves and the world. Through the process of recalling and reviewing memories, personal meaning and internal standards of worth could be derived, challenges that had been faced and overcome could be identified and drawn on for self-confidence and inspiration, and a cohesive and integrated understanding of the self could be formed on the basis of significant events and experiences.

Overall, we found CRT to be a mode of treatment that was useful for young adults with depressive symptoms. It is hoped that the preceding discussion helps to illuminate the use of CRT, both in general and with younger adults, and highlights its merit as a psychotherapeutic approach to alleviating depression. 


\section{References}

Arnett, J. J. (2000) Emerging adulthood. A theory of development from the late teens through the twenties. American Psychologist, 55, 469-80. doi:10.1037//0003066X.55.5.469.

Arnett, J. J. (2004). Emerging adulthood: The winding road from late teens through the twenties. New York: Oxford University Press.

Beck, A. T., Rush, A. J., Shaw, B. F., \& Emery, G. (1979). Cognitive therapy of depression. New York, NY: Guildford Press.

Billings, A. G., \& Moos, R. H. (1985). Psychosocial stressors, coping and depression. In E. E. Beckman \& W. R. Leber (Eds.), Handbook of depression: Treatment, assessment, and research (pp. 940-974). Homewood, IL: Dorsey Press.

Bluck, S., \& Alea, N. (2008). Remembering being me: The self continuity function of autobiographical memory in younger and older adults. In F. Sani (Ed.), Self Continuity: Individual and Collective Perspectives (55-70). New York: Psychology Press.

Bluck, S., \& Alea, N. (2009). Thinking and talking about the past: Why remember? Applied Cognitive Psychology, 23, 1089-1104. doi:10.1002/acp.1612

Butler, R. N. (1974). Successful aging and the role of the life review. Journal of the American Geriatrics Society, 22, 529-535. Retrieved from http://psycnet. apa.org.ezproxy-m.deakin.edu.au/psycinfo/1975-09317-001

Cappeliez, P. (2002). Cognitive-reminiscence therapy for depressed older adults in day hospital and long-term care. In Webster, J. D. and Haight, B. K. (eds), Critical 
Advances in Reminiscence Work: From Theory to Application. Springer, New York, $300-313$.

Cappeliez, P., \& Robitaille, A. (2010). Coping mediates the relationships between reminiscence and psychological well-being amongst older adults. Aging and Mental Health, 14, 807-818. doi:10.1080/13607861003713307

Carstensen, L. L. (2006). The influence of a sense of time on human development. Science, 312, 1913-1915. doi:10.1126/science.1127488

Erikson, E. H. (1980). Identity and the Life Cycle. New York: Norton.

Fromholt, P., Larsen, P., \& Larsen, S. F. (1995). Effects of late-onset depression and recovery of autobiographical memories. Journal of Gerontology, 50, 74-81. doi:10.1093/geronb/50B.2.P74

Habermas, T., Ott, L. M., Schubert, M., Schneider, B., \& Pate, A. (2008). Stuck in the past: Negative bias, explanatory style, temporal order, and evaluative perspectives in life narratives of clinically depressed individuals. Depression and Anxiety, 25, E121-E132. doi:10.1002/da.20389

Hallford, D. J. (2014). Cognitive-reminiscence therapy for the treatment of depression in young adults (Unpublished doctoral dissertation). Deakin University, Melbourne, Australia.

Halford, D. J., \& Mellor, D. (2013a). Reminiscence-based therapies for depression: Should they be used only with older adults? Clinical Psychology: Science and Practice, 20, 452-468. doi:10.1111/cpsp.12043 
Hallford, D. J., \& Mellor, D. (2013b). Cognitive-reminiscence therapy and usual care for depression in young adults: Study protocol for a randomized controlled trial. Trials, 14:343. doi:10.1186/1745-6215-14-343

Hallford, D. J., \& Mellor, D. (2016). Autobiographical memory-based intervention for depressive symptoms in young adults: A randomized controlled trial of cognitivereminiscence therapy. Psychotherapy and psychosomatics, 85(4), 246-249. doi: $10.1159 / 000444417$

Hallford, D. J., Mellor, D., \& Burgat, M. E. (2018). A Qualitative Study of Young Adults Experience With a Reminiscence-Based Therapy for Depressive Symptoms. Emerging Adulthood. doi:10.1177/2167696818769175

Hallford, D. J., Mellor, D., \& Cummins, R. A. (2013): Adaptive autobiographical memory in younger and older adults: The indirect association of integrative and instrumental reminiscence with depressive symptoms, Memory, 21, 444-457. doi:10.1080/09658211.2012.736523

Hammen, C. (2005). Stress and depression. Annual Review of Clinical Psychology, 1, 293-319. doi:10.1146/annurev.clinpsy.1.102803.143938

Lang, F. R., \& Carstensen, L. L. (2002). Time counts: future time perspective, goals, and social relationships. Psychology and Aging, 17(1), 125-139. doi:10.1037/08827974.17.1.125

Liu, X., Li, L., Xiao, J., Yang, J., \& Jiang, X. (2012). Abnormalities of autobiographical memory of patients with depressive disorders: A meta-analysis. Psychology and Psychotherapy: Theory, Research and Practice, 86, 353-373. doi:10.1111/j.20448341.2012.02077.x 
Korte, J., Westerhof, G. J., \& Bohlmeijer, E. T. (2012). Mediating processes in an effective life-review intervention. Psychology and Aging, 27, 1172-1181. doi: $10.1037 / \mathrm{a} 0029273$

Korten, N., Comijs, H. C., Lamers, F., \& Penninx, B. W. (2012). Early and late onset depression in young and middle aged adults: Differential symptomatology, characteristics and risk factors? Journal of affective disorders, 138, 259-267. doi:10.1016/j.jad.2012.01.042.

Kuwabara, S. A., Van Voorhees, B. W., Gollan, J. K., \& Alexander, G. C. (2007). A qualitative exploration of depression in emerging adulthood: disorder, development, and social context. General Hospital Psychiatry, 29, 317-324. doi:10.1016/j.genhosppsych.2007.04.001.

McAdams, D. P. (2001). The psychology of life stories. Review of General Psychology, 5(2), 100-122. doi:10.1037/1089-2680.5.2.100

Mossakowski, K. N. (2011). Unfulfilled expectations and symptoms of depression among young adults. Social Science \& Medicine, 73, 729-736. doi:10.1016/j.socscimed. 2011.06.021

Neshat-Doost, H. T., Dalgleish, T., Yule, W., Kalantari, M., Ahmadi, S. J., Dyregrov, A., \& Jobson, L. (2013). Enhancing autobiographical memory specificity through cognitive training: An intervention for depression translated from basic science. Clinical Psychological Science, 1(1), 84-92. doi:10.1177/2167702612454613

Nezu, A. M. (2004). Problem-solving and behavior therapy revisited. Behavior Therapy, 35, 1-33. doi:10.1016/S0005-7894(04)80002-9 
Pinquart, M., Forstmeier, S. (2012). Effects of reminiscence interventions on psychosocial outcomes: A meta-analysis. Aging and Mental Health, 16, 541-558. doi:10.1080/13607863.2011.651434

Raes, F., Williams, J. M. G., \& Hermans, D. (2009). Reducing cognitive vulnerability to depression: A preliminary investigation of MEmory Specificity Training (MEST) in inpatients with depressive symptomatology. Journal of Behavior Therapy and Experimental Psychiatry, 40(1), 24-38. doi:10.1016/j.jbtep.2008.03.001

Serrano, J. P., Latorre, J. M., Gatz, M., \& Montanes, J. (2004). Life review therapy using autobiographical retrieval practice for older adults with depressive symptomatology. Psychology and Aging, 19, 272-277. doi:10.1037/08827974.19.2.272

Serrano Selva, J. P., Latorre Postigo, J. M., Segura L. R., Bravo, B. N., Córcoles, M. J. A., López, M. N., Trives, J. J. R., \& Gatz, M. (2012). Life review therapy using autobiographical retrieval practice for older adults with clinical depression. Psicothema, 24(2), 224-229. Retrieved from http://156.35.33.98/reunido/index. php/PST/article/viewFile/9613/9358

Singer, J. A., Blagov, P., Berry, M., \& Oost, K. M. (2013). Self-defining memories, scripts, and the life story: Narrative identity in personality and psychotherapy. Journal of Personality. doi:10.1111/jopy.12005

Sumner, J. A., Griffith, J. W., \& Mineka, S. (2010). Overgeneral autobiographical memory as a predictor of the course of depression: A meta-analysis. Behavior Research and Therapy, 48, 614-625. doi:10.1016/j.brat.2010.03.013 
Watt, L. M., \& Cappeliez, P. (1995). Reminiscence interventions for the treatment of depression in older adults. In B.K. Haight \& J. D. Webster (Eds.), The Art and Science of Reminiscing: Theory, Methods, and Applications (pp. 221-232).

Washington, DC: Taylor and Francais.

Watt. L. M., \& Cappeliez, P. (2000). Integrative and instrumental reminiscence therapies for depression in older adults. Intervention strategies and treatment effectiveness. Aging and Mental Health, 4, 166-177. doi:10.1080/13607860050008691

Webster, J. D., \& Ma, X. (2013). A balanced time perspective in adulthood: Well-being and developmental effects. Canadian Journal on Aging, 32(4). doi:10.1017/S0714980813000500

Westerhof, J. D., Bohlmeijer, E. T., \& Webster, J. D. (2010). Reminiscence and mental health: a review of recent progress in theory, research and interventions. Aging and Society, 30, 697-721. doi:10.1017/S0144686X09990328

Westerhof, J. D., Bohlmeijer, E. T., van Beljouw, I. M. J., \& Pot, A. M. (2010). Improvement in personal meaning mediates the effects of a life review intervention on depressive symptoms in a randomised controlled trial. The Gerontologist, 50, 541-549. doi:10.1093/geront/gnp168

White, M., \& Epston, D. (1990). Narrative means to therapeutic ends. New York: Norton.

Williams, J. M. G., Barnhofer, T., Crane, C., Hermans, D., Raes, F., Watkins, E., \& Dalgeish, T. (2007). Autobiographical memory specificity and emotional disorder. Psychological Bulletin, 133, 122-148. doi:10.1037/0033-2909.133.1.122 
Wong, P. T. P. (1995). The processes of adaptive reminiscence. In B. K. Haight \& J.D. Webster (Eds.). The art and science of reminiscing: Theory, methods, and applications (pp. 23-35). Washington, DC: Taylor and Francais.

Zisook, S., Lesser, I., Stewart, J. W., Wisniewski, S. R., Balasubramani, G. K., Fava, M., . . Rush, A. J. (2007). Effect of age at onset on the course of major depressive disorder. American Journal of Psychiatry, 164, 1539-1546. doi:10.1176/appi.ajp. 2007.06101757 
Third level: Broader life narratives that cohesively link experiences together

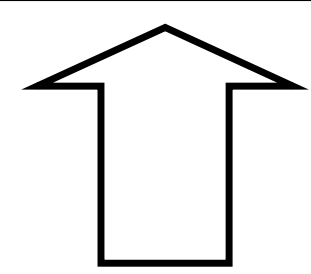

Second level: Appraisals of the general significance of events and experiences

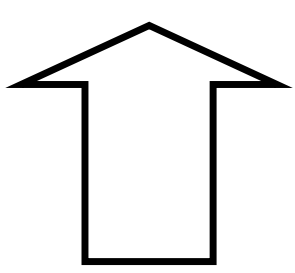

First level: Facts, emotions, and thoughts relating to specific memories

Figure 1. A three-level model of analysis in CRT. 
Third level: Challenges and that I have met and risen to over my life, that have shown me I am a capable person, and can often overcome difficulties when I need and want to

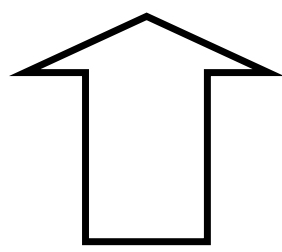

Second level:

Moving as a period of change, which required me to learn new things, take risks, and find ways to open up to others

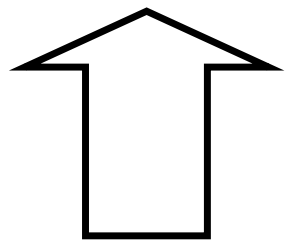

First level:

Moving to another country with different cultural conventions. Felt anxious, isolated, and overwhelmed. Thought that things would not be as good as before, and I wouldn't be able to make friends
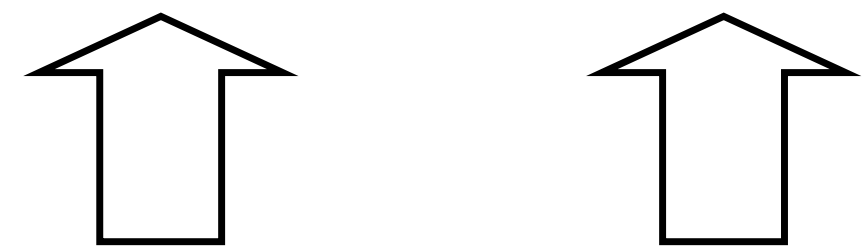

Second level:

An experience that made me feel autonomous, capable, and able to do things without support from my parents.

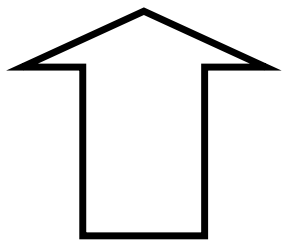

First level:

Buying my first car, and having to work different jobs to pay it off. Felt worried in case I couldn't pay it off, but proud that I stuck at it. Thought that this was a sign of my independence
Second level:

An achievement that took a lot of work and perseverance, but was fun

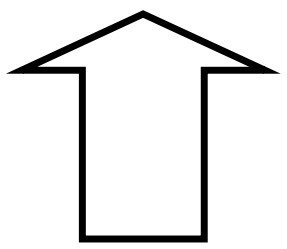

First level: Working hard in my football team to get to the grand finals, and remembering when I found out we had made it. Felt excited and proud. Thought that this was payoff for all the training and matches

Figure 2. An example of a three-level model of analysis in CRT drawing on several specific autobiographical memories. 
Table 1.

Common Life Events and Issues that Emerged Through Reminiscence with Young Adults

\begin{tabular}{|c|c|c|c|c|}
\hline Family & Social & Occupational & Educational & Identity \\
\hline Changing & Learning how & Hobbies and & Starting/finishing & Emerging \\
\hline relationships & to make & interests & primary school, & worldviews and \\
\hline \multirow[t]{4}{*}{ with parents } & friends, and & pursued in a & high school and & changes in \\
\hline & identifying & club or group, & university & thinking on \\
\hline & valued traits in & or individually & & socio-political \\
\hline & people & & & issues \\
\hline Growing apart & Falling in and & Winning or & Studying for & Looking further \\
\hline or closer with & out of love for & achieving in & exams and & into one's \\
\hline \multirow[t]{3}{*}{ siblings } & the first time & sports, artistic & completing & future and what \\
\hline & & endeavours etc. & tertiary-level & it might hold \\
\hline & & & assignments & \\
\hline Moving out of & Reflecting on & Securing a first & Developing & Consolidating \\
\hline the family & long-lasting, & job & specialised & interests over \\
\hline \multirow[t]{4}{*}{ home } & and "best- & & knowledge in an & time, and \\
\hline & friend" & & area & integrating \\
\hline & relationships & & & them into the \\
\hline & Changes in & Gaining & Building & $\begin{array}{l}\text { self-concept } \\
\text { Gaining legal }\end{array}$ \\
\hline \multirow{4}{*}{$\begin{array}{l}\text { Desire to make } \\
\text { parents proud, } \\
\text { and fulfil their } \\
\text { expectations }\end{array}$} & social circles & meaningful & professionalism & status and \\
\hline & and social & employment & through study & increased \\
\hline & systems over & & & autonomy and \\
\hline & time & & & responsibility \\
\hline
\end{tabular}

